Article

\title{
Improving Liquid Entry Pressure of Polyvinylidene Fluoride (PVDF) Membranes by Exploiting the Role of Fabrication Parameters in Vapor-Induced Phase Separation VIPS and Non-Solvent-Induced Phase Separation (NIPS) Processes
}

\author{
Faisal Abdulla AlMarzooqi, Muhammad Roil Bilad and Hassan Ali Arafat * \\ Department of Chemical and Environmental Engineering, Masdar Institute of Science and Technology, \\ P.O. Box 54224 Abu Dhabi, UAE; falmarzooqi@masdar.ac.ae (F.A.A.); roilbilad130@yahoo.com (M.R.B.) \\ * Correspondence: harafat@masdar.ac.ae; Tel.: +971-2-810-9119
}

Academic Editor: Enrico Drioli

Received: 25 December 2016; Accepted: 4 February 2017; Published: 14 February 2017

\begin{abstract}
Polyvinylidene fluoride (PVDF) is a popular polymer material for making membranes for several applications, including membrane distillation (MD), via the phase inversion process. Non-solvent-induced phase separation (NIPS) and vapor-induced phase separation (VIPS) are applied to achieve a porous PVDF membrane with low mass-transfer resistance and high contact angle (hydrophobicity). In this work, firstly, the impacts of several preparation parameters on membrane properties using VIPS and NIPS were studied. Then, the performance of the selected membrane was assessed in a lab-scale direct-contact MD (DCMD) unit. The parametric study shows that decreasing PVDF concentration while increasing both relative humidity $(\mathrm{RH})$ and exposure time increased the contact angle and bubble-point pore size (BP). Those trends were investigated further by varying the casting thickness. At higher casting thicknesses and longer exposure time (up to $7.5 \mathrm{~min}$ ), contact angle (CA) increased but BP significantly decreased. The latter showed a dominant trend leading to liquid entry pressure (LEP) increase with thickness.
\end{abstract}

Keywords: membrane distillation; polyvinylidene fluoride; hydrophobic; non-solvent-induced phase separation; vapor-induced phase separation

\section{Introduction}

The membrane distillation (MD) process is driven by the vapor pressure difference between a hot and a cold stream in the feed and permeate side of a membrane module, respectively. This driving force is created by the temperature difference between the hot and cold streams. Both streams are separated by a membrane that ideally features the following properties: high hydrophobicity, high porosity, low tortuosity, and low thickness. In addition to low mass transfer resistance, an MD membrane should be able to withstand a certain liquid entry pressure (LEP) to avoid wetting [1]. Theoretical LEP of a membrane is a function of maximum pore size (also called bubble point, BP) and surface hydrophobicity, expressed in terms of the surface contact angle (CA) with water. LEP can be estimated using the Cantor-Laplace equation [1].

Polyvinylidene fluoride (PVDF) is an attractive MD membrane material, which can be made into membranes via phase inversion. It has low surface energy, good thermal stability and low conductivity. In the phase inversion process, a thermodynamically-stable polymer solution (mixture of polymer and its solvent) is brought to instability by several means: non-solvent-induced phase separation (NIPS), vapor-induced phase separation (VIPS) or temperature-induced phase separation (TIPS). All of these 
have been applied to prepare PVDF-based MD membranes [2-4]. The NIPS process is the most popular and has been used to prepare different types of membranes, including reverse osmosis, nanofiltration, ultrafiltration, microfiltration and MD [5,6]. When used to produce PVDF membranes in a strong solvent/non-solvent system, typically, the NIPS process is characterized by a relatively fast mass transfer rate (instantaneous demixing, relative to VIPS) leading to asymmetric structure (dense top layer, supported by a more porous layer) [5], narrow pore size distribution (PSD), relatively low mean flow pore size (MPS) and maximum pore size (BP) very close to MPS; all are desired properties of an MD membrane. However, under those same conditions, NIPS also produces a membrane with a fairly smooth surface (low roughness) leading to a low surface energy (high CA), which promotes low LEP and thus membrane wetting.

Increasing the CA of PVDF membranes has been demonstrated by applying weak non-solvent (ethanol) in a single bath system [7] and weak non-solvent (ethanol) as the first bath followed by strong non-solvent (water) in the second bath of a dual-bath coagulation system [8]. These methods increased the CA significantly (up to $153^{\circ}$ ) but the resulting membranes had low porosity originating from a dense structure as a result of a delayed demixing that led to a high mass-transfer resistance. Superhydrophobic PVDF membranes can also be obtained via the VIPS process, but feature large $\mathrm{BP}$ that diminishes the LEP [8]. A study which investigated the phase inversion mechanisms that led to the formation of a highly hydrophobic surface [3] emphasized the importance of solution composition pathway when crossing both binodal and spinodal lines in the three-phase diagram. Depending on the route of the composition changes, porous and spherulitic structures resulting from PVDF crystallization resulted in a hydrophobic surface, while net-like morphology as a result of spinodal-decomposition (SD) resulted in a superhydrophobic surface [8]. The latter can be produced under low PVDF concentrations $(<5 \%)$, high relative humidity $(\mathrm{RH})$ and high vapor temperature. However, too-low PVDF concentrations produce membranes that are vulnerable to wetting and low-salt rejections (SR). At such low concentrations, there is a lack of mechanical stability in the membrane and the anchoring of the membrane on the support layer becomes problematic. To avoid this problem, Fan et al. [9] applied the co-casting technique, consisting of high $(16 \% w / w)$ and low $(10 \% w / w)$ PVDF concentrations at the bottom and top layer, respectively, to achieve a highly hydrophobic surface by ensuring low PVDF concentration on the top of the film.

In this study, the interplays between surface and bulk membrane properties on one hand and the fabrication parameters during phase inversion, on the other hand, are disentangled by systematically exploiting the role of VIPS and NIPS parameter variations on the membrane characteristic. This study is distinguished from previous studies in trying to relate changes in surface properties to parameters in the membrane fabrication process, through a systematic disentanglement of the latter. It utilizes the fact that the water vapor absorption/imbibition rates are much faster than water/solvent diffusion rates, resulting in a gradient of composition profile across the casting film thickness (being the lowest at the top of film) during film exposure to humid atmosphere [10]. The surface structure mixture of nano- and micro-scale roughness [7], which promotes higher hydrophobicity, is expected. Meanwhile, this surface structure maintains a pore structure that controls salt rejection. Membrane formation mechanism via VIPS was first studied, followed by a series of tests to investigate the impact of polymer concentration, relative humidity $(\mathrm{RH})$ and exposure time. The role of thickness was then investigated, followed by a moisture uptake test as a way to experimentally support our findings. Finally, the performance of the selected membrane was assessed in a lab-scale direct-contact MD (DCMD) unit.

\section{Materials and Methods}

\subsection{Membrane Preparation}

All membranes were prepared using PVDF polymer (HSV900, Mw 92,840 kDa, Arkema, Colombes, France), dimethylacetamide (DMAC, Sigma-Aldrich, St. Louis, NA, USA) and deionized (DI) water as the polymer, solvent and non-solvent, respectively. In the NIPS process, after being 
thoroughly dissolved and degassed, the polymer solution was cast on a non-woven support (NWS) (Novatexx 2471, donated by Freudenberg-filter, Weinheim, Germany) at $24{ }^{\circ} \mathrm{C}$ using a doctor blade with adjustable height to give a wet-casting thickness of 250, 500, 750 or $1000 \mu \mathrm{m}$ (henceforth referred to as the "casting thickness"). This was immediately followed by immersion in a coagulation bath containing deionized water. A number of membrane samples were prepared via a VIPS process which was similar to the NIPS process except that it involved film exposure to humid air at different percentages of relative humidity (RH): $37 \%, 60 \%$ and $80 \%$. This exposure was after the casting step, for a certain time (henceforth called "exposure time") followed by immersion into a coagulation bath (DI water at room temperature). The details of preparation parameters for each sample are shown in Table 1.

Table 1. Summary of membrane preparation parameters.

\begin{tabular}{cc}
\hline Parameter & Range \\
\hline Polyvinylidene fluoride (PVDF) concentration & $8 \%, 10 \%, 12 \%$ and $15 \%(w / w)$ \\
Relative humidity & N/A $*, 37 \%$ (room relative humidity), $60 \%$ and $80 \%$ \\
Exposure time & $0 * 2,5,10,30,60$ and $120 \mathrm{~min}$ \\
Casting thickness & $250,500,750$ and $1000 \mu \mathrm{m}$ \\
\hline${ }^{*}$ Not Applicable: For non-solvent-induced phase separation (NIPS) process.
\end{tabular}

\subsection{Membrane Characterization}

The microstructure of the membrane surface was observed using scanning electron microscopy (SEM, Quanta-250, FEI, Hillsboro, OR, USA). Samples were gold and palladium-coated at $50 \AA$ thickness prior to SEM analysis. The MPS, BP and PSD were measured using a capillary flow porometer (CFP, Porous Materials Inc., Ithaca, NY, USA). Volume porosity was calculated experimentally using a gravimetric method using Galwick ${ }^{\circledR}$ as a wetting liquid. The CA of DI water on the membrane and NWS surfaces was measured using the sessile drop method with a contact angle goniometer (Krüss DSA $10 \mathrm{Mk2}$, Hamburg, Germany) at $24^{\circ} \mathrm{C}$. Multiple measurements (at least six) at different locations of the same membrane sample were taken to enhance data accuracy.

\subsection{Moisture Uptake Test}

The cast film on the NWS was placed on a digital mass balance in a controlled humidity chamber. The evolution of weight was measured and recorded over time. The tests were performed for the three applied RHs, 37\%, 60\% and 80\%. This test works on the assumption that the evaporation rate of DMAC is very low, i.e., negligible compared to the water vapor imbibition rates into the cast film. This assumption is based on the fact that there is an overall increase in the membrane weight with time during the water vapor imbibition stage. It is also worth noting that water is much more volatile than DMAC (water and DMAC vapor pressures are $3167 \mathrm{~Pa}$ and $557 \mathrm{~Pa}$, respectively, at $25^{\circ} \mathrm{C}$ ) $[11,12]$.

\subsection{DCMD Experiments}

DCMD experiments were carried out under controlled conditions using a custom built lab-scale DCMD setup. The description of the set-up and its operation can be found elsewhere [13]. The feedand permeate-side liquids were $35 \mathrm{~g} / \mathrm{L} \mathrm{NaCl}$ solution and DI water, respectively. Both were circulated at a constant flow rate of $25 \mathrm{~L} / \mathrm{h}$ (corresponding to a linear flow velocity of $0.08 \mathrm{~m} / \mathrm{s}$ in the DCMD chamber). During each experiment $(\sim 3 \mathrm{~h})$, the feed and permeate temperatures were maintained at 70 and $25^{\circ} \mathrm{C}$, respectively. Permeate overflow was continuously measured by means of a microbalance and the membrane flux was calculated from this value.

The salt rejection (SR) was calculated based on the following formula:

$$
\mathrm{SR}(\%)=\left(1-\frac{C_{\text {Permeate }}}{C_{\text {Feed }}}\right) \times 100
$$


where $C_{\text {feed }}$ and $C_{\text {permeate }}$ are the salt concentrations in the feed and permeate streams, respectively $(\mathrm{g} / \mathrm{L})$.

\section{Results and Discussion}

\subsection{Membrane Morphologies in NIPS vs. VIPS}

Two distinct membrane morphologies were obtained for membranes prepared using NIPS and VIPS processes. In general, VIPS membranes, irrespective of applied RH, show a much higher CA $\left(>125^{\circ}\right)$ than the NIPS membrane $\left(\mathrm{CA}=90.74^{\circ}\right)$, as shown in Table 2. This finding regarding VIPS morphology was also reported elsewhere [14]. When calculating the LEP of membranes prepared using both processes, they all range from 103 to $107 \mathrm{kPa}$, which makes the membranes vulnerable to wetting. This low LEP range, mostly caused by the high BP values for all membranes, disallows the application of high operational pressure (typically up to $200 \mathrm{kPa}$ depending on MD module system and desired cross-flow velocity) that is required to overcome pressure-drop in both the feed and the permeate streams in a module system. Additional pressure is also required to induce sufficient cross-flow velocity for controlling concentration polarization on the membrane surface. The advantage of high CA from the VIPS and low BP from NIPS to achieve high LEP is diminished because of large BP $(10-21 \mu \mathrm{m})$ of VIPS membranes and low CA of NIPS membranes. Nevertheless, this finding brings about a potential for disentangling these features in the two processes to gain the advantages of both processes (high CA and low BP). As mentioned at the bottom of Table 2, all the LEP values in this table and throughout this manuscript were calculated using the Cantor-Laplace equation,

$$
\mathrm{LEP}=\frac{-2 \gamma \cos \theta}{r_{\max }}
$$

where $\gamma$ is the surface tension of the wetting liquid (in this case water at $25^{\circ} \mathrm{C}, 0.07199 \mathrm{~N} / \mathrm{m}$ ), $\theta$ is the contact angle between the membrane and the wetting liquid (water) and $r_{\max }$ is the maximum pore radius of the membrane (i.e., bubble-point pore size). It is worth noting that the assumption in this equation is that pores are perfectly cylindrical with a constant radius of curvature.

Table 2. Summary of membrane properties prepared using NIPS and vapor-induced phase separation (VIPS) process, prepared under casting thickness of $500 \mu \mathrm{m}$ using 12\% w/w PVDF concentration.

\begin{tabular}{ccccc}
\hline Membrane & MPS $(\boldsymbol{\mu m})$ & BP $(\boldsymbol{\mu m})$ & CA & LEP $(\mathbf{k P a}){ }^{*}$ \\
\hline NIPS & 0.08 & 0.38 & 90.7 & 103.91 \\
VIPS $(37 \%$ RH $)$ & 7.80 & 12.25 & 136.3 & 106.79 \\
$(60 \%$ RH $)$ & 5.85 & 21.19 & 130.9 & 103.56 \\
$(80 \%$ RH $)$ & 6.07 & 10.63 & 127.2 & 106.55 \\
\hline
\end{tabular}

* Calculated using Cantor-Laplace equation. MPS: mean flow pore size; BP: bubble-point pore size; CA: contact angle; LEP: liquid entry pressure; RH: relative humidity.

Since all membranes were prepared from the same material, the difference in the surface morphology can thus be held responsible for the change in surface CA values, as can be seen in Table 2 and Figure 1. It is known that highly hydrophobic surface is a result of the presence of multi-scale surface features consisting of both micro- and nano-scale roughness [7,15-17], also known as the lotus leaf effect. The high contact angle on the surface of a lotus leaf is a consequence of such micro- and nano-scale roughness, the micro- and nano-scale roughness can be clearly seen in our case for VIPS- $60 \%$ and VIPS- $80 \%$ SEM images, Figure 1 . The micron-sized pore mouth most likely contributed to the micro-scale roughness for both VIPS and NIPS membranes. The higher CA of the VIPS membranes originated from the nano-scale roughness from nodule-like morphology (circled features in Figure 1) that most probably originated from small spherulites of PVDF crystals. These can be clearly seen in the VIPS-60\% and VIPS- $80 \%$ SEM images. 
NIPS
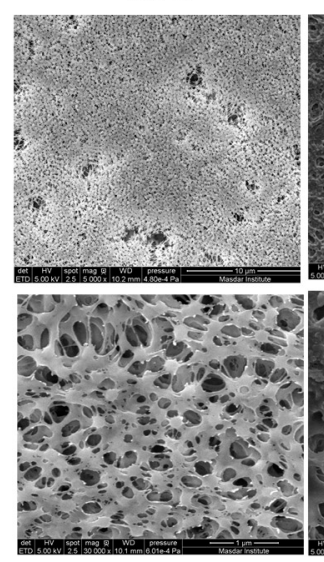

VIPS-37\%
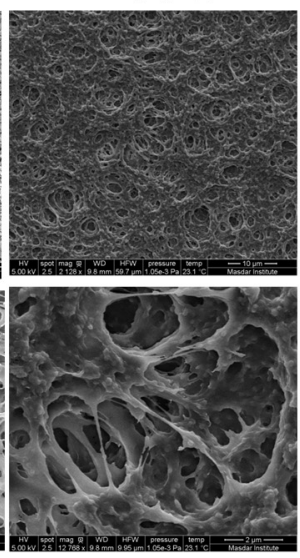

VIPS-60\%
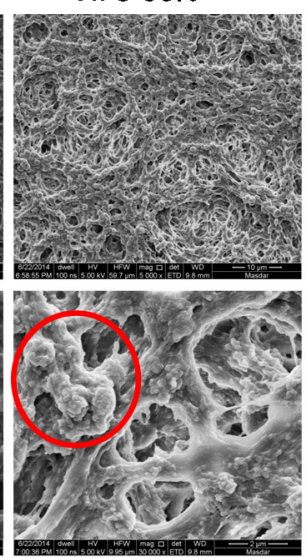

VIPS-80\%
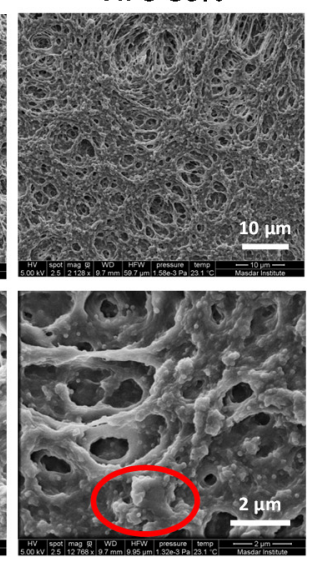

Figure 1. Top surface scanning electron microscope (SEM) images of membranes prepared using NIPS (non-solvent-induced phase separation) and VIPS (vapor-induced phase separation) processes with $12 \% w / w$ PVDF (Polyvinylidene fluoride) concentration and cast thickness of $500 \mu \mathrm{m}(10 \mu \mathrm{m}$ and $2 \mu \mathrm{m}$ scale bares for all top and bottom row SEM images, respectively).

The formation of more crystalline structure in the VIPS membranes was possible due to the nature of compositional change pathway during the phase inversion process. This pathway can be explained using a three-component system phase diagram (Figure 2). Starting with a NIPS process (path D), spontaneous demixing occurs immediately once the polymer solution gets in contact with the non-solvent in the coagulation bath [18-20]. In this case, the rate of non-solvent intrusion into the casted polymer film is much higher than the rate of solvent extrusion, leading to highly porous membrane as discussed in detail by Khayat and Matsuura (2011). As the compositional pathway moves from the initial polymer solution composition to the final membrane composition, it enters the two-phase region where it splits into polymer-rich (pint 1, Figure 2) and lean (point 2, Figure 2) phases connected by a tie-line. As the final membrane composition is approached, both solvent and non-solvent eventually evaporate during the drying period and the membrane becomes a single-phase polymer solid. At the final membrane composition, the two phases are at thermodynamic equilibrium. A polymer-rich phase with composition $S$ is marked in Figure 2 as well as a polymer-lean phase with composition L. Point $L$ represents the composition of the voids or pores of the membrane which contain water which eventually evaporate during the drying stage. At the other extreme, when a VIPS process is applied without the final coagulation bath step, delayed demixing leads the compositional pathway to follow path A (Figure 2) [21]. In this case, the rate of non-solvent intrusion into the casted polymer film is much slower than the rate of solvent extrusion. This path represents the case of lowest RH, where the composition of the polymer solution never crosses the binodal curve and maintains a single phase. As solvent and non-solvent exchange occurs at a rate much slower than in the NIPS case, there are three different mass-transfer processes occurring simultaneously; water vapor is absorbed into the polymer solution while DMAC and water evaporate, leaving the polymer solution. The former absorption rate of water is relatively much higher than the evaporation as will be discussed later (Section 3.3). The driving force for the water absorption in this case is the chemical potential difference between the atmosphere surrounding the polymer solution and the polymer solution itself. Over time, the difference between these two chemical potentials decreases, causing the rate of water vapor absorption to change as the membrane goes through the compositional pathway A. The decrease in chemical potential is depicted in the compositional pathway of A by the increasing slope of the path, representing a decreasing intake of water and a sharper movement towards the PVDF-rich phase. As the composition crosses the crystallization line, the polymer solution solidifies, forming crystal nuclei and eventually forming a membrane with crystalline structures (evidence of this is discussed later, Figure 6). Compositional paths B-F and C-E (Figure 2) represent a combination of 
a complete NIPS (pathway D) and a complete VIPS (pathway A) process [21,22]. As the RH increases, the compositional path moves from pathway A towards D, with B-F and C-E representing two RH cases with B-F having a lower RH than C-E. As the RH increases, the chemical potential difference between the polymer solution and the atmosphere surrounding it increases as well, leading to a higher rate of absorption of water (as can be seen later in Figure 8). Pathway B-F starts in a similar way to path A, but with a higher RH. As the polymer solution loses DMAC and gains water it enters the meta-stable region [21]. Once there, the casted film is immersed in the coagulation bath. At this point the pathway shifts from B to F, following a path similar to D but at a different initial point in the two-phase region. Ultimately, the membrane forms with different surface morphology, pore properties and porosity [18]. Pathway $\mathrm{C}$ is similar to $\mathrm{B}$ but with an even higher $\mathrm{RH}$ and a different exposure time. Here, the membrane is immersed in the coagulation bath after it crosses the spinodal line [21] moving from path $\mathrm{C}$ to $\mathrm{E}$.

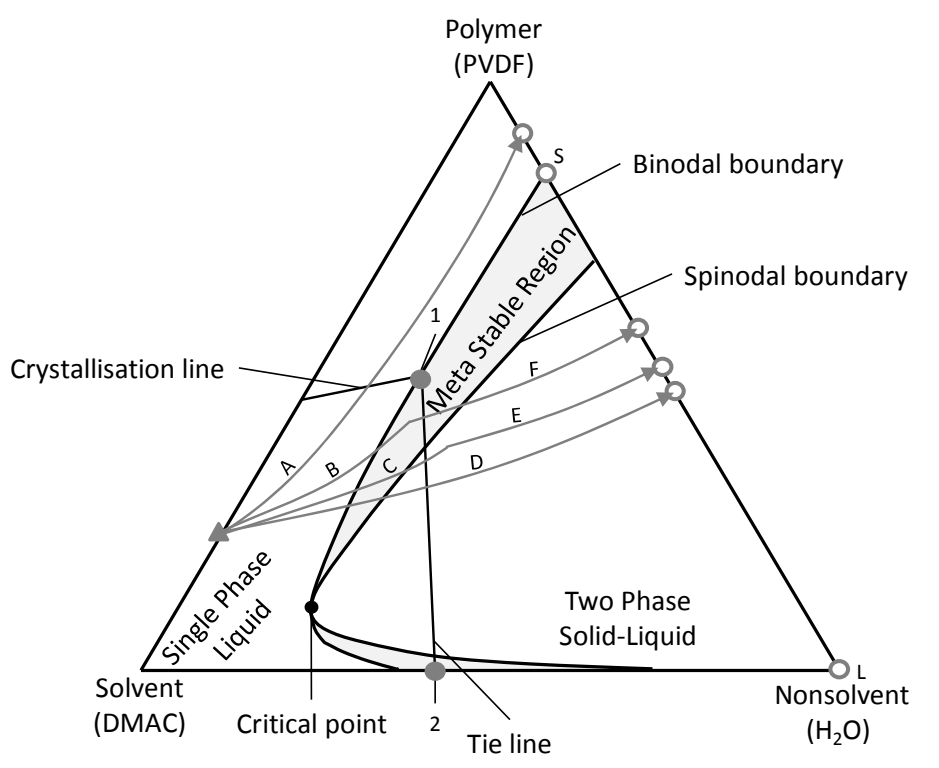

Figure 2. A three-component phase diagram for the polymer, solvent and non-solvent system generally depicting the VIPS and NIPS processes. Path A and D represent delayed (VIPS) and spontaneous (NIPS) de-mixing, respectively. Paths $B$ and $C$ represent an initial VIPS process followed by a NIPS. $\Delta$ is initial polymer solution composition and $\mathrm{O}$ is composition of final membrane. DMAC: dimethylacetamide.

Unlike the NIPS process, in which introducing the cast film into the coagulation bath promotes instantaneous demixing (Figure 1), slow uptake of water from humid air allows the composition change to cross or approach the crystallization region in the phase diagram (Figure 2) and form crystalline morphology, allowing the crystal nuclei to grow thanks to the semi-crystalline property of PVDF, as observed in the case of VIPS-37\%, VIPS- $60 \%$ and VIPS- $80 \%$ in Figure 1 [23]. This way, crystal nuclei were formed and they had sufficient time to grow and produce a spherulitic structure in the resulting membrane as illustrated in Figure 1. The large PVDF agglomerates are highly crystalline spherulites (micron size), while the nodule-like morphologies over the surface of the spherulites are the ends of the PVDF polymer chains. This pathway also alters the membrane pore size to be much larger, as shown in Figure 3. On one hand, the rapid demixing process disallows further growth of the polymer-lean phase, resulting in small pore size of NIPS membranes [24]. On the other hand, slow uptake of water from humid air gives sufficient time for the polymer-lean hase for growth and coalescence after the composition enters the meta-stable region and before phase inversion is completed, creating the large pores in the VIPS-made membranes. 

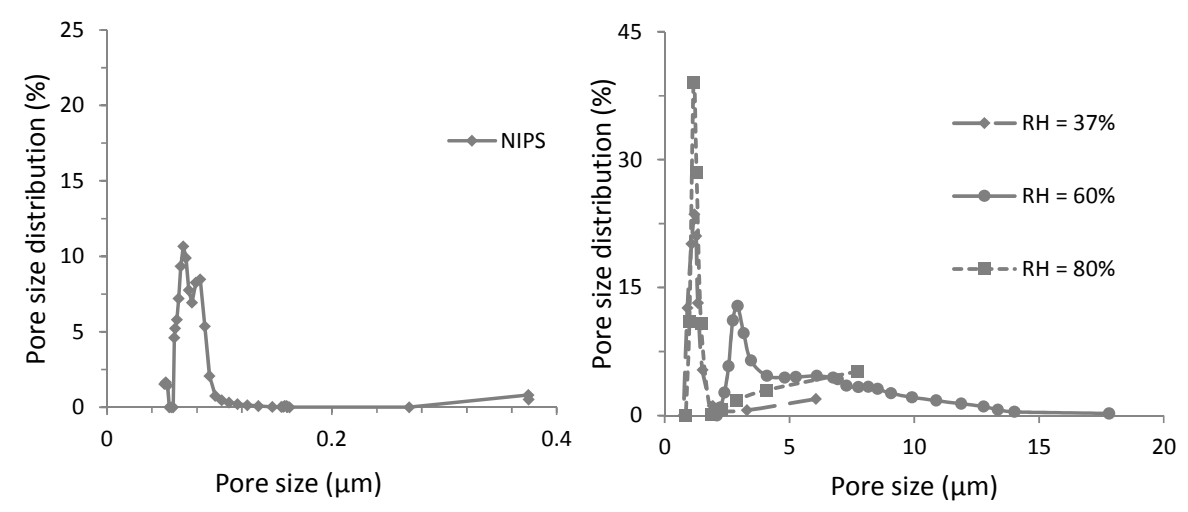

Figure 3. Pore size distributions (PSD) of membranes made via the NIPS (left) and VIPS (right) processes. Casting thickness: $500 \mu \mathrm{m}$, polymer solution: $12 \% w / w$ PVDF.

\subsection{Going from NIPS to VIPS: A Parametric Study}

As an attempt to combine the advantages of both VIPS and NIPS processes, a series of parametric studies to understand the role of several parameters on structure, CA and BP, were performed. The main objective is to obtain membranes which are highly porous with a surface morphology in favor of high CA, while keeping an acceptable BP $(0.4-0.6 \mu \mathrm{m})$.

\subsubsection{Effect of PVDF Concentration}

Polymer concentration plays a significant role in determining the demixing process. Lower polymer concentrations are more likely to experience spinodal-decomposition (SD), producing a net-like surface structure in favor of the superhydrophobic property because their initial compositions are closer to the critical point in which binodal and spinodal lines are in a very close proximity [5]. Indeed, it was found that PVDF concentration affected membrane surface morphology, as shown in Figure 4. However, a net-like structure associated with superhydrophobicity originating from SD was not observed for all membrane samples prepared in this study. It was only observed in the $8 \%$ case as can be seen in the inset of the $8 \%$ membrane in Figure 4 . This is mainly because of relatively high applied concentrations of PVDF compared to the one applied by [5]. Net-like structure is expected only during SD at very low PVDF concentrations and under high casting temperature $\left(60^{\circ} \mathrm{C}\right)$ and high $\mathrm{RH}(100 \%)$. Nevertheless, a low degree of spherulitic morphology coupled with a large number of nano-scale nodules (Figure 4) was observed for $8 \%$ PVDF solution, leading to a high CA of $123^{\circ}$ (Table 3).

As PVDF concentration increased, a decrease in both surface porosity as well as mean pore size was observed (Table 3). The change in PVDF concentrations clearly affected the hydrophobicity (CA) of the membranes. As the concentration increased, the CA decreased. This can be attributed to the flat morphology at a higher concentration which led to almost hydrophilic membranes (CA of 15\% PVDF was below $90^{\circ}$ ).

The advantage of membranes prepared from lower PVDF concentrations is diminished by their large BP and MPS (Table 3). Therefore, the obtained LEP data suggests that a desirable PVDF concentration is around $12 \%$. Membranes prepared using lower PVDF concentrations exhibit hydrophobic properties thanks to rough surface structure, but feature a rather large BP. On the other hand, the membrane made from a higher PVDF concentration $(15 \%)$ had a very flat surface that reduced its CA, but offered a substantially low BP. As a result, by merely tailoring PVDF concentration, an optimum concentration can be ball-parked with respect to contact angle and pore sizes. In this case, the value is $12 \%$. 
Table 3. Properties of membranes cast using PVDF solutions of different concentrations at cast thickness: $500 \mu \mathrm{m}$, relative humidity (RH): 60\% and exposure time: $30 \mathrm{~min}$.

\begin{tabular}{cccccc}
\hline PVDF Concentration $(w / w)$ & MPS $(\mu \mathbf{m})$ & $\mathbf{B P}(\mu \mathrm{m})$ & $\mathbf{C A}\left({ }^{\circ}\right)$ & LEP $(\mathbf{k P a})$ & Volume Porosity \\
\hline $8 \%$ & 0.2076 & 7.5956 & 123.2 & 108.30 & $48 \%$ \\
$10 \%$ & 0.1196 & 4.0325 & 113.1 & 111.21 & $70 \%$ \\
$12 \%$ & 0.089 & 0.210 & 94.6 & 143.93 & $88 \%$ \\
$15 \%$ & 0.064 & 0.652 & 88.9 & 96.64 & $67 \%$ \\
\hline
\end{tabular}
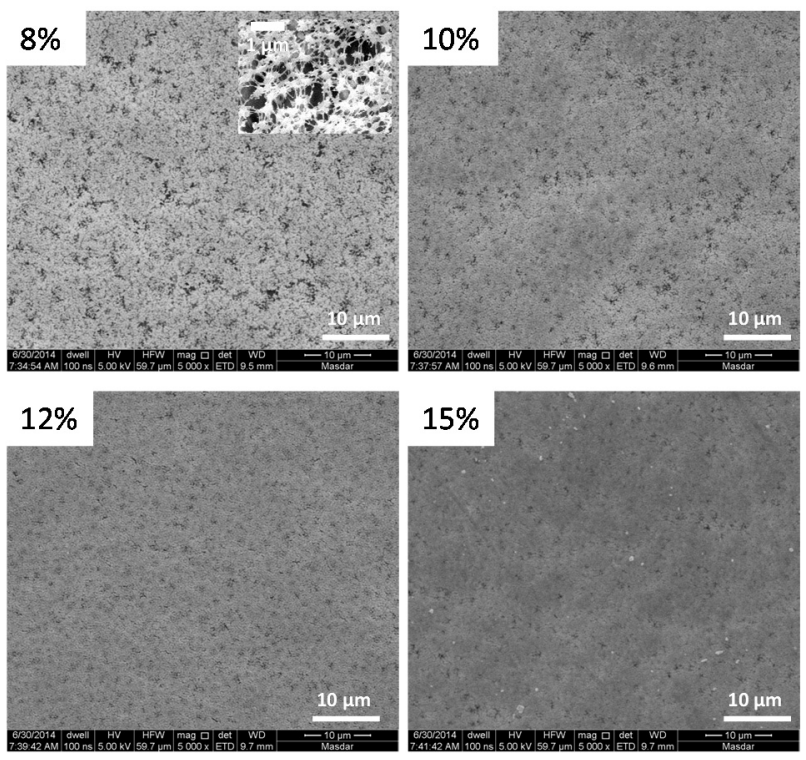

Figure 4. Surface SEM images of membrane samples prepared using polymer solutions of different PVDF concentrations (casting thickness: $500 \mu \mathrm{m}, \mathrm{RH}$ : 60\%, exposure time: $30 \mathrm{~min}$ ).

\subsubsection{Effect of Humidity}

Higher RH provides larger chemical potential for water to absorb on the cast film. Therefore, for a similar period of time, a higher amount of water uptake is expected for a higher $\mathrm{RH}$ (later discussed in Section 3.3). By considering the high miscibility of water and DMAC, the transport of DMAC from the deeper zone of the cast film to the top of the film and influx of water from the top of the film is expected, leading to lower concentration of PVDF at the top of the film. This is due to the transport of DMAC from deep in the bulk of the casted film to the top layer, leaving behind a PVDF-rich phase at the bottom layers of the cast film and a PVDF-lean phase at the top layers. Increasing humidity and/or exposure time thus promotes a higher concentration gradient of PVDF that promotes a change of surface hydrophilicity, like the one observed as an effect of PVDF concentration. This is in agreement with the obtained CA results seen in Table 4. CA increased from $91.0^{\circ}$ to $104.5^{\circ}$ and $93.6^{\circ}$ to $98.2^{\circ}$ by increasing exposure time from 2 to $10 \mathrm{~min}$ for $\mathrm{RHs}$ of $60 \%$ and $80 \%$, respectively.

As the RH and exposure time increased, the presence of droplet-like pore mouths on the membrane surface increased (see SEM images of $80 \%$ at $10 \mathrm{~min}$, Figure 5), which led to the creation of larger pores, especially at higher RH. These observations are in agreement with BP and MPS results, both of which increased with an increase in RH (Table 4). It is worth noting that some large pores on the membrane surface were a result of localized water vapor condensation on the top of the polymer film that was unavoidable sometimes during RH tests. Whenever the ambient humidity was below the RH set-point, the humidifier created extra water vapor that could potentially precipitate on the top of polymer film, leading to local concentration of water. 
Table 4. Membranes properties with casting thickness of $500 \mu \mathrm{m}$ and PVDF concentration of $12 \%(w / w)$.

\begin{tabular}{cccccc}
\hline RH $(\%)$ & Exposure Time $(\mathbf{m i n})$ & MPS $(\mu \mathrm{m})$ & BP $(\mu \mathrm{m})$ & CA $\left({ }^{\circ}\right)$ & LEP $(\mathbf{k P a})$ \\
\hline- & 2 & 0.057 & 0.10 & 91.0 & 118.34 \\
$60 \%$ & 5 & 0.060 & 0.13 & 94.3 & 167.66 \\
- & 10 & 0.137 & 0.78 & 104.5 & 137.18 \\
- & 2 & 0.073 & 0.17 & 93.6 & 141.17 \\
$80 \%$ & 5 & 0.126 & 0.85 & 97.7 & 108.86 \\
- & 10 & 0.140 & 2.90 & 98.2 & 105.66 \\
\hline
\end{tabular}

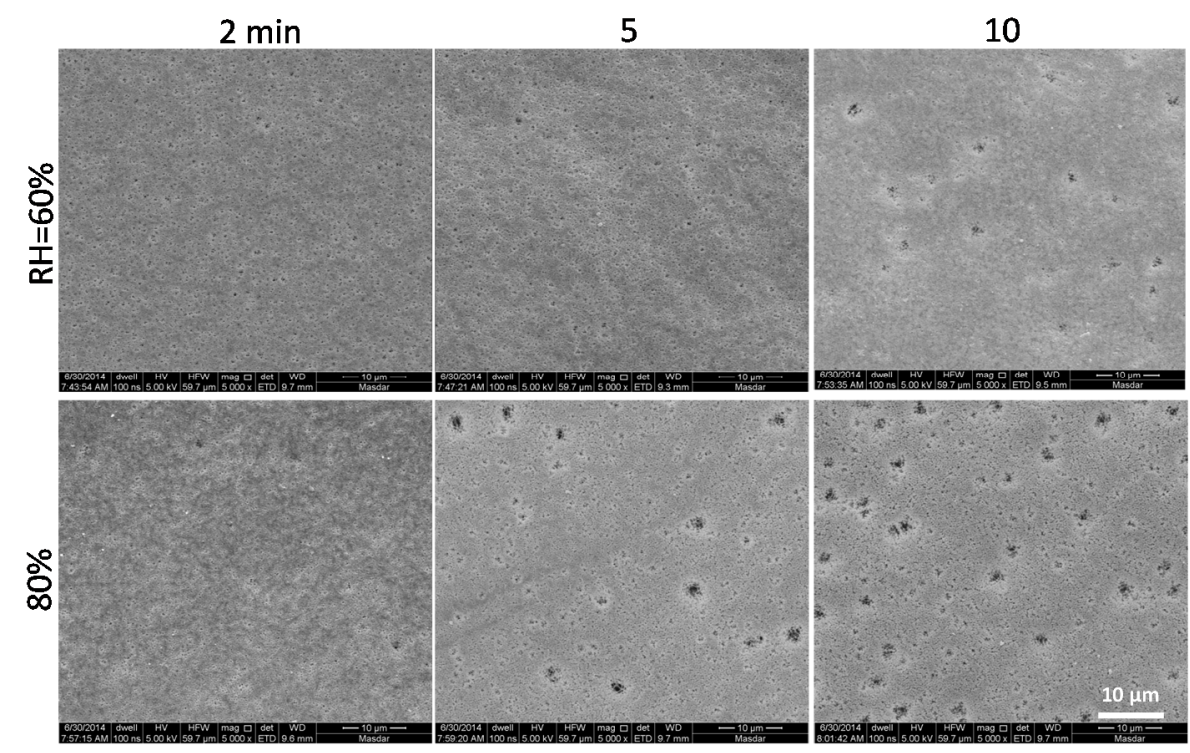

Figure 5. Surface SEM images of membrane samples prepared under different $\mathrm{RH}$ and different exposure times. Casting thickness: $500 \mu \mathrm{m}$, polymer solution: 12\% $w / w$ PVDF.

\subsubsection{Effect of Exposure Time}

A clear gradual change of the surface structure of membranes prepared using $12 \% \mathrm{PVDF}, 60 \% \mathrm{RH}$ and casting thickness of $500 \mu \mathrm{m}$ is shown in Figure 6. This change translated into the in trend of CA shown in Figure 7A. A drastic change of surface structure was observed at exposure time between 1 and $2 \mathrm{~h}$, in which the structure changed from a flat surface to a spherulitic-like structure (Figure 6). This structure is responsible for the substantial increase in CA up to $137^{\circ}$. However, the increase in CA was also mirrored by an increase in BP (Figure 7), which diminished the LEP value. The LEP data shows a gradual decrease with exposure time (Table 4), which is in agreement with the SEM and the BP results (Figures 6 and 7).

Increasing exposure time enlarged the membranes' MPS, which reached a plateau at $1 \mathrm{~h}$, then jumped substantially at $2 \mathrm{~h}$ (Figure 7B). This is mainly because of the drastic change of membrane structure discussed above. Also, significant increase of BP only occurred after a certain exposure time (30 $\mathrm{min}$ ). These findings suggest that extending the exposure time can significantly change the surface hydrophobicity without substantially changing the BP. In other words, it is possible to increase the LEP and MPS, which implies a larger MD flux at an assumed constant pore density, by extending the exposure time. 


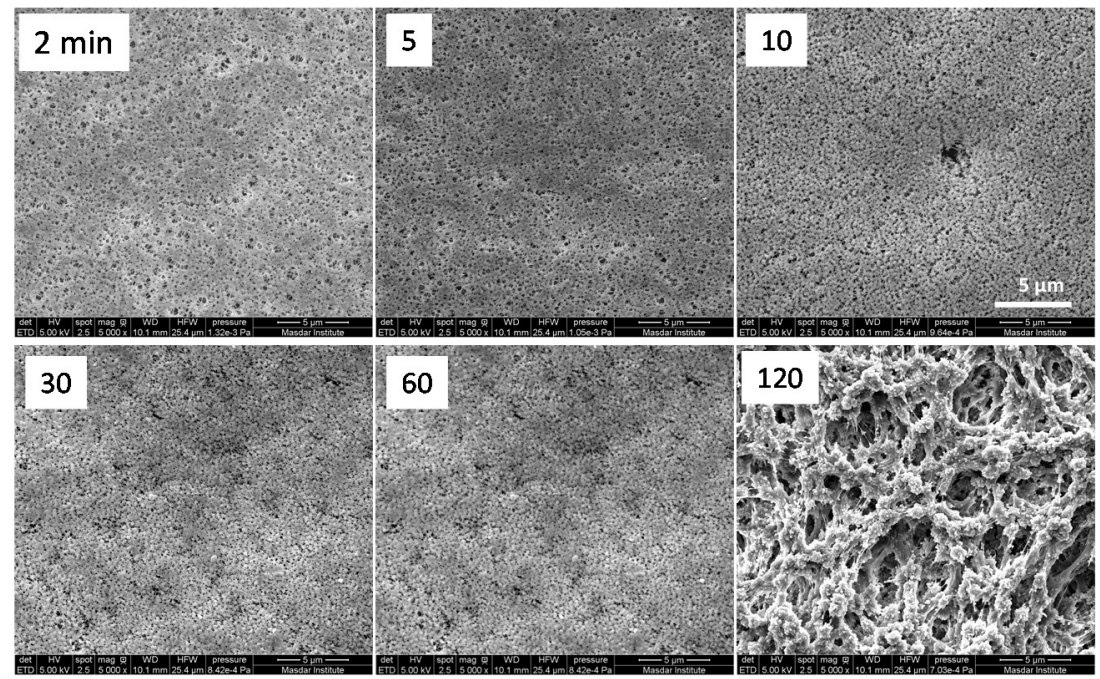

Figure 6. Surface SEM images of membrane samples prepared at different exposure times under $60 \%$ $\mathrm{RH}, 12 \% w / w$ PVDF concentration and 500- $\mu \mathrm{m}$ casting thickness. All images have the same scale bar, shown in the top-right image.

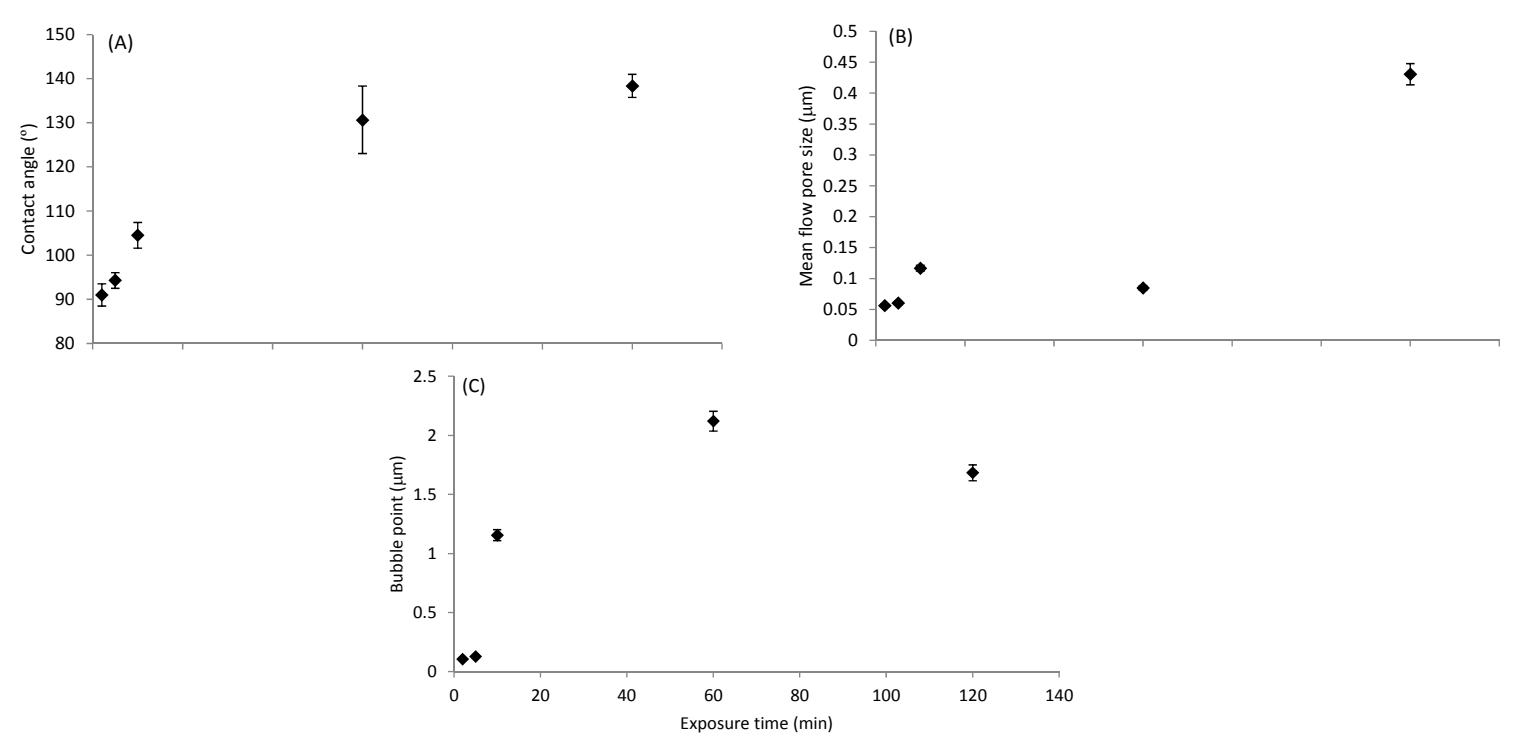

Figure 7. Impact of exposure time on (A) CA (contact angle); (B) MPS (mean flow pore size) and (C) BP (bubble-point pore size), for membranes prepared under $\mathrm{RH}$ of $60 \%$ and at casting thickness of $500 \mu \mathrm{m}$ with $12 \% w / w$ PVDF concentration.

\subsection{Moisture Uptake during the VIPS Process}

Figure 8 shows mass variation of polymer cast film during exposure to different humidity values $(37 \%, 60 \%$ and $80 \% \mathrm{RH})$. In all cases, soon after the exposure started, the film mass started to increase until it reached a maximum value, and then it decreased. As the $\mathrm{RH}$ increased, a shift in the maximum uptake was observed, peaking at around 20,90 and $450 \mathrm{~min}$ for 37\%, 60\% and 80\% RH, respectively. The rate of moisture uptake before reaching the maximum value was also faster for higher RH. Two competing mass transfer phenomena in the cast film are expected during the exposure: (1) the water flows into the film due to the hygroscopic and miscible nature of water and DMAC; and (2) the outflow of DMAC and water from the cast film. Before reaching the maximum mass, water uptake was a predominant phenomenon, leading to an increase in mass of the cast film. The increase in absorption rate of water at higher $\mathrm{RH}$ was a result of a larger chemical potential at higher water 
concentration in air. After reaching the maximum value, solvent and water evaporation from the casted film prevailed over water sorption, resulting in a weight loss. The water sorption stopped when there was no chemical potential difference, which occurred when the amount of water on the cast film was large enough. The loss of mass then continued as a result of the concomitant DMAC and water loss from the film.

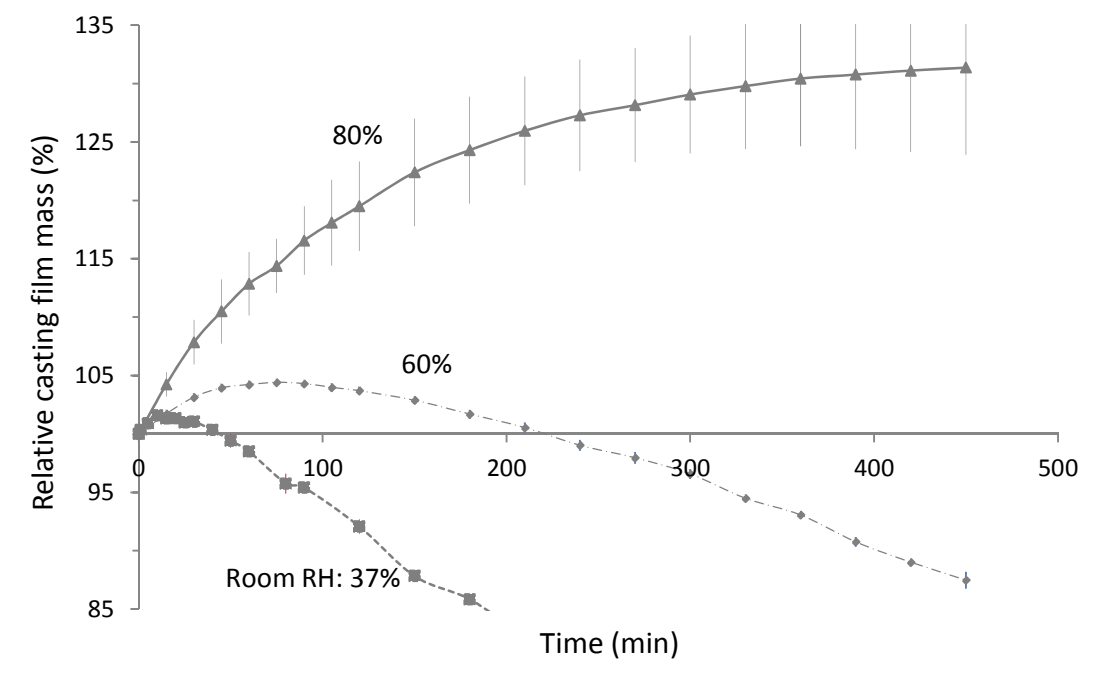

Figure 8. Water uptakes from humid atmosphere at different relative humidity $(\mathrm{RH})$ values $(37 \%, 60 \%$ and $80 \%$ ) for membranes prepared using $12 \% w / w$ PVDF solution at a casting thickness of $500 \mu \mathrm{m}$.

The water sorption mechanism explained earlier allows the understanding of mass transfer phenomenon within the cast film. Just after the exposure started, at the very top of the film, a local exchange of water and DMAC occurred. This process continued further at deeper locations within the film creating a change in composition across the film thickness, as explained using a phenomenological model by [25]. This process would continue as long as the top film layer did not solidify as a result of phase separation. However, in the case of PVDF/DMAC/water system, during the humid air exposure, a thin film of water was observed on the top of the cast film after certain time, which was more obvious for higher RH ( $60 \%$ and $80 \%$ ) as also found by [26] for a poly(ether-imide) / $N$-methylpyrolidone/water system. This finding suggests that the phase inversion of the top film layer had started and completed earlier, creating a dense barrier for further exchange of DMAC and water at the deeper location. Consequently, concentration gradient from top (being the lowest PVDF concentration) to the bottom (being the highest) could not be achieved fully. This explains why the increment of surface hydrophobicity only occurred within certain period of time (2-10 $\mathrm{min}$ ) (Figure 7). Within this time interval and until just before phase separation completed, a rapid composition change within the cast film was still possible, resulting in a change in surface properties [10]. Up to this point, it is not clear why further exposure time beyond $1 \mathrm{~h}$ resulted in a shift of surface morphology as shown in Figure 6 .

\subsection{Decoupling Pore Size and Surface Properties: Effect of Casting Thickness}

The three main parameters explored in Section 3.2 and the phenomenological explanation of water imbibition and water/DMAC mass transfer in Section 3.3 are important to (1) determine the properties of the resulting membrane and (2) the suitability of the membrane for MD application. In an attempt to elucidate the roles of those parameters and the underlying mechanisms to produce desired MD membranes, the impact of casting thickness was further explored. Our key hypothesis here is that the gradient in polymer concentration during the phase inversion process can be exploited by membrane thickness control, to obtain highly hydrophobic surface properties while maintaining an acceptable BP and MPS. The tests were performed under $80 \% \mathrm{RH}$, which offers higher chemical potential of water absorption into the cast film. 
A gradual change in the surface morphology of the membrane samples was observed by extending the exposure time (Figure 9), which was similar to the one observed by varying the time parameter at $\mathrm{RH}$ of $60 \%$ in Figure 6. However, no obvious impact of casting thickness was observed, or at least it was not clearly visible from the surface SEM images. The change of CA with casting thickness shows a general decrease and this is clearly observed in the case of 7.5-min exposure time (Figure 10). It is also observed that the effect of casting thickness on CA and BP is more pronounced in the cases of relatively longer exposure times ( 5 and $7 \mathrm{~min}$ ) in comparison to shorter ones ( 2 and $3.5 \mathrm{~min}$ ). These observations may be attributed to the influx and outflux of water vapor and DMAC, respectively. At a thinner casting thickness, the resistance to mass transfer of water vapor and DMAC is relatively lower than that at a thicker casting thickness. Consequently, and due to this larger influx and outflux in the case of thinner casting thickness, the morphological change (and consequently roughness) is more pronounced, leading to higher contact angles. The consequences of lower mass transfer resistance in thinner membranes leading to larger influx and outflux of water vapor and DMAC, respectively, can explain the general decrease of BP with thickness. As more water vapor is absorbed by the cast film, more DMAC is partitioned out, leaving behind large void spaces and consequently pores. This effect is less prominent in thicker membranes, causing lower BP due to the higher mass transfer resistance to water vapor influx. In fact, these observations reconfirm the findings on the effect of increasing humidity on BP (Table 4). As was seen before, the BP increased with humidity due to the higher chemical potential between the cast solution and humid atmosphere. Therefore, by decreasing the mass transfer resistance or increasing the chemical potential (humidity), a larger BP is obtained. As a consequence of the CA and BP trends, the LEP values in Figure 10 show a slight increase in LEP with thickness and this is clearly seen for the case of the 5-min exposure time. In this case, the slight increase in LEP can potentially be attributed to the relatively larger percentage decrease in BP in comparison to the decrease in CA. As shown in Figure 10, the casting thickness played a significant role in maintaining a small BP. As the casting thickness was reduced, the incremental BP size significantly increased, which substantially diminished the LEP. Under this situation, increasing thickness offers substantial advantages since under thicker casting thickness (e.g., $1000 \mu \mathrm{m}$ ) LEP could be controlled well.

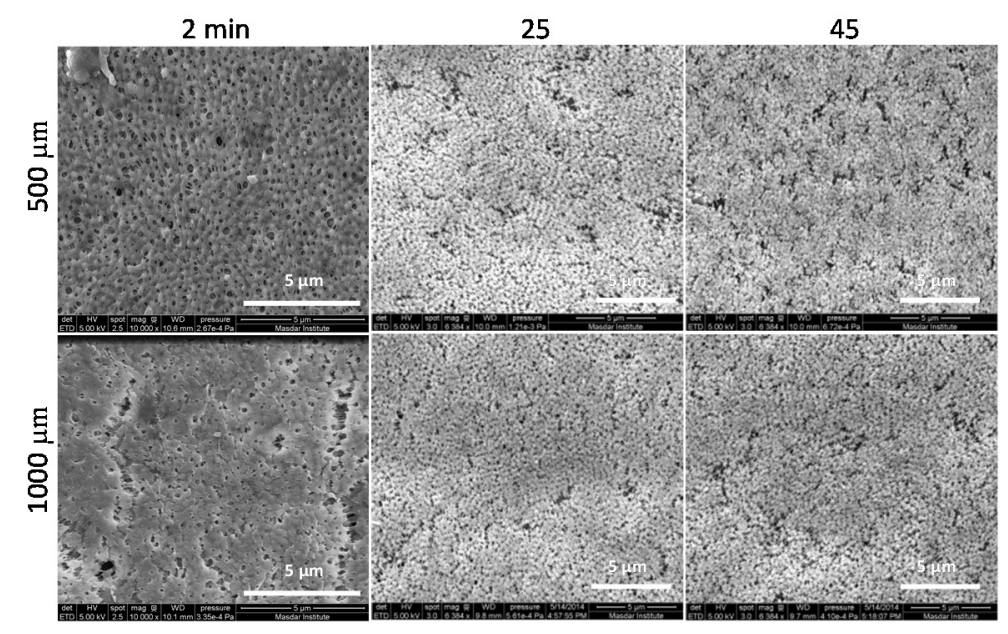

Figure 9. Surface SEM images of PVDF membranes prepared using VIPS process at different casting thicknesses and exposure times, all using 12\% $w / w$ PVDF polymer solution and under $80 \% \mathrm{RH}$. 

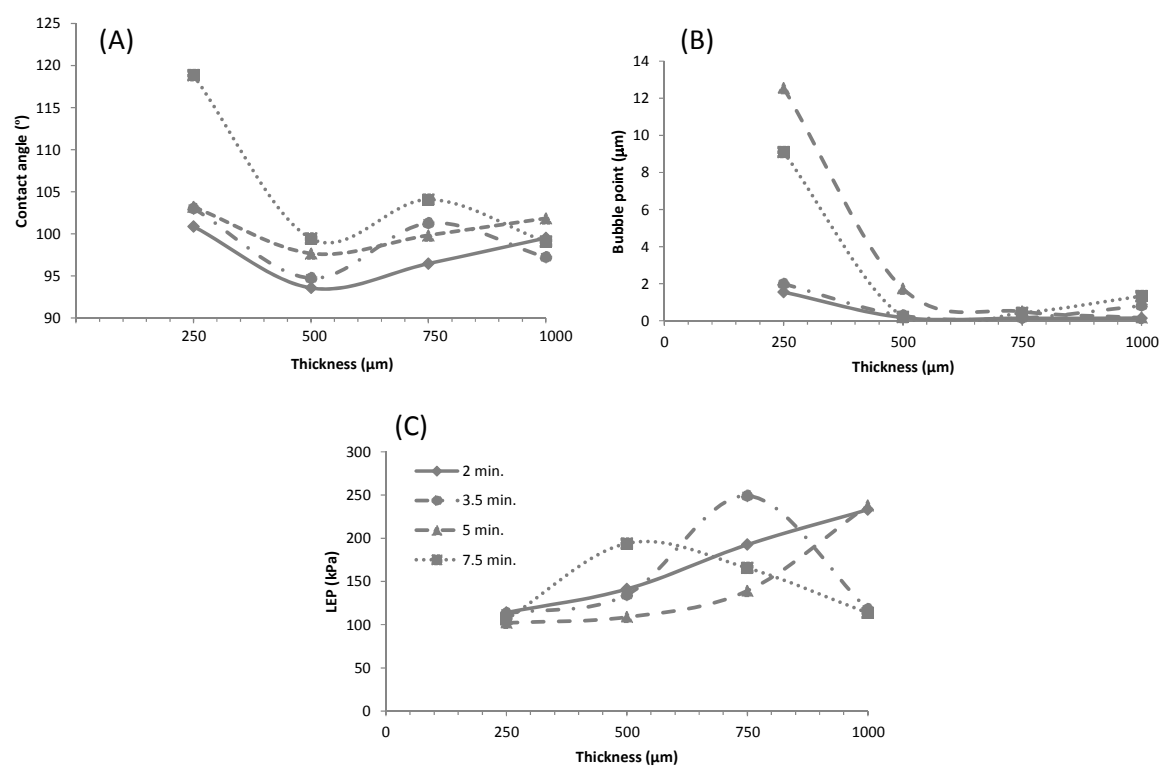

Figure 10. Impact of casting thickness on the properties of PVDF membrane prepared using VIPS process, all using 12\% $w / w$ PVDF polymer solution and under $80 \%$ RH. (A) Contact angle profile; (B) Bubble point profile; (C) LEP profile.

\subsection{Performance}

DCMD performance tests were performed for selected membranes with varying casting thicknesses (500 and $1000 \mu \mathrm{m}$ ) and exposure times (5, 10 and $15 \mathrm{~min}$ ). These particular membranes were selected because they offered an acceptable range of contact angle and pore sizes (Table 5). The results (Table 5) showed a flux range of $9-32 \mathrm{~L} / \mathrm{m}^{2} \cdot \mathrm{h}$ and SR $>99 \%$. These flux values are within the range of MD flux reported for PVDF membranes [27-29]. In general, no obvious trend could be extracted from the salt rejection data since they are considerably high for all samples. When comparing the impact of casting thickness, at exposure time of 5 and $10 \mathrm{~min}$, the flux of the 1000- $\mu \mathrm{m}$ membrane was higher than the 500- $\mu \mathrm{m}$ one, while the opposite trend was encountered at the 15-min exposure time. The trend of flux with exposure time for both casting thicknesses was also different. For the $500-\mu \mathrm{m}$ thickness, the flux changed with exposure time: 8.75 (at $5 \mathrm{~min}$ ), 13.77 (at $10 \mathrm{~min}$ ) and $22.19 \mathrm{~L} / \mathrm{m}^{2} \cdot \mathrm{h}$ (at $15 \mathrm{~min}$ ). Meanwhile, for the $1000-\mu \mathrm{m}$ membranes, the flux values were 27.76 , increasing to 31.82 and then dropping to 10.98 for exposure times of 5, 10 and $15 \mathrm{~min}$, respectively. The trend of flux values unfortunately cannot be very well explained with the existing data in this study. It is not a function of MPS, nor a function casting thickness. In addition to surface morphology and MPS, it is most likely that the VIPS process also alters other properties (e.g., tortuosity, pore density, surface porosity or other) that affect the mass transfer resistance of the resulting membranes. Nevertheless, the ability to obtain DCMD membranes having an acceptable rejection and flux while improving their LEP has been well proven in this study.

Table 5. Summary of direct-contact membrane distillation (DCMD) performance using membrane samples fabricated at $80 \% \mathrm{RH}$ and $12 \% w / w$ PVDF casting solution.

\begin{tabular}{|c|c|c|c|c|c|c|}
\hline $\begin{array}{c}\text { Casting } \\
\text { Thickness }(\mu \mathrm{m})\end{array}$ & $\begin{array}{l}\text { Exposure } \\
\text { Time (min) }\end{array}$ & $\begin{array}{c}\text { Average Flux } \\
\left(\mathrm{L} / \mathrm{m}^{2} \cdot \mathrm{h}\right)\end{array}$ & $\begin{array}{c}\text { Average Salt } \\
\text { Rejection (SR) (\%) }\end{array}$ & $\begin{array}{l}\text { Porosity } \\
\text { (\%) }\end{array}$ & MPS ( $\mu \mathrm{m})$ & $\begin{array}{c}\text { Net Thickness } \\
(\mu \mathrm{m})\end{array}$ \\
\hline \multirow{3}{*}{500} & 5 & $8.7 \pm 3.2$ & 99.95 & 71 & 0.0863 & 246 \\
\hline & 10 & $13.8 \pm 7.0$ & 99.75 & 87 & 0.2347 & 265 \\
\hline & 15 & $22.2 \pm 6.9$ & 99.91 & 76 & 0.1649 & 294 \\
\hline \multirow{3}{*}{1000} & 5 & $27.8 \pm 1.9$ & 99.81 & 65 & 0.0693 & 307 \\
\hline & 10 & $31.8 \pm 2.9$ & 99.91 & 68 & 0.0641 & 327 \\
\hline & 15 & $11.0 \pm 7.5$ & 99.99 & 66 & 0.068 & 381 \\
\hline
\end{tabular}




\section{Conclusions}

Through a study of VIPS and NIPS processes, it has been shown that the VIPS process is an effective method to obtain a PVDF membrane with high contact angle and low BP, through manipulation of casting thickness. The membrane properties were tuned in this study by exploiting the role of casting thickness to obtain a membrane with a high CA, low BP and acceptable DCMD flux and SR. The parametric study results show that decreasing the PVDF concentration of the polymer cast solution and increasing RH and exposure time increased CA, MPS and BP. Those trends were exploited further by varying the casting thickness. At higher casting thicknesses and longer exposure times, CA increased but BP significantly decreased, while the latter showed a more dominant trend leading to an optimum condition with respect to maximum LEP. This was obtained in this study at a higher casting thickness $(1000 \mu \mathrm{m}$, thanks to lower BP) and short exposure time ( $5 \mathrm{~min}$, thanks to higher CA). Moisture uptake experiments showed peaks at which the mass transfer mechanisms shifted from being dominated by water vapor absorption to evaporation of DMAC and water. This allowed the determination of the ideal exposure time to obtain the desired membrane hydrophobicity and pore size. The SR and DCMD fluxes of the selected membrane samples showed that they are within the value ranges of PVDF membranes reported for MD in literature, demonstrating a substantial gain in LEP without sacrificing flux, or rejection.

Acknowledgments: This work was funded by the Cooperative Agreement between the Masdar Institute of Science and Technology, Abu Dhabi, UAE and the Massachusetts Institute of Technology (MIT), Cambridge, MA, USA, Reference No. 02/MI/MI/CP/11/07633/GEN/G/00.

Author Contributions: F.A.A., M.R.B. and H.A.A. conceived and designed the experiments; F.A.A. and M.R.B. performed the experiments; F.A.A., M.R.B. and H.A.A. analyzed the data; H.A.A. contributed reagents/materials/analysis tools; F.A.A., M.R.B. and H.A.A. wrote the paper.

Conflicts of Interest: The authors declare no conflict of interest.

\section{References}

1. Warsinger, D.M.; Swaminathan, J.; Guillen-Burrieza, E.; Arafat, H.A.; Lienhard, J.H., V. Scaling and fouling in membrane distillation for desalination applications: A review. Desalination 2015, 356, 294-313. [CrossRef]

2. Mu, C.; Su, Y.; Sun, M.; Chen, W.; Jiang, Z. Fabrication of microporous membranes by a feasible freeze method. J. Membr. Sci. 2010, 361, 15-21. [CrossRef]

3. Peng, Y.; Fan, H.; Ge, J.; Wang, S.; Chen, P.; Jiang, Q. The effects of processing conditions on the surface morphology and hydrophobicity of polyvinylidene fluoride membranes prepared via vapor-induced phase separation. Appl. Surf. Sci. 2012, 263, 737-744. [CrossRef]

4. Simone, S.; Figoli, A.; Criscuoli, A.; Carnevale, M.C.; Rosselli, A.; Drioli, E. Preparation of hollow fibre membranes from PVDF/PVP blends and their application in VMD. J. Membr. Sci. 2010, 364, 219-232. [CrossRef]

5. Peng, Y.; Fan, H.; Dong, Y.; Song, Y.; Han, H. Effects of exposure time on variations in the structure and hydrophobicity of polyvinylidene fluoride membranes prepared via vapor-induced phase separation. Appl. Surf. Sci. 2012, 258, 7872-7881. [CrossRef]

6. Devi, S.; Ray, P.; Singh, K.; Singh, P.S. Preparation and characterization of highly micro-porous PVDF membranes for desalination of saline water through vacuum membrane distillation. Desalination 2014, 346, 9-18. [CrossRef]

7. Peng, M.; Li, H.; Wu, L.; Zheng, Q.; Chen, Y.; Gu, W. Porous poly(vinylidene fluoride) membrane with highly hydrophobic surface. J. Appl. Polym. Sci. 2005, 98, 1358-1363. [CrossRef]

8. Kuo, C.-Y.; Lin, H.-N.; Tsai, H.-A.; Wang, D.-M.; Lai, J.-Y. Fabrication of a high hydrophobic PVDF membrane via nonsolvent induced phase separation. Desalination 2008, 233, 40-47. [CrossRef]

9. Fan, H.; Peng, Y.; Li, Z.; Chen, P.; Jiang, Q.; Wang, S. Preparation and characterization of hydrophobic PVDF membranes by vapor-induced phase separation and application in vacuum membrane distillation. J. Polym. Res. 2013, 20, 134. [CrossRef] 
10. Matsuyama, H.; Teramoto, M.; Nakatani, R.; Maki, T. Membrane formation via phase separation induced by penetration of nonsolvent from vapor phase. I. Phase diagram and mass transfer process. J. Appl. Polym. Sci. 1999, 74, 159-170. [CrossRef]

11. Felder, R.M.; Rousseau, R.W. Elementry Principles of Chemical Processes, (with CD); John Wiley \& Sons: Hoboken, NJ, USA, 2008.

12. Gopal, R.; Rizvi, S. Vapour pressures of some mono-and di-alkyl substituted aliphatic amides at different temperatures. J. Indian Chem. Soc. 1968, 45, 13-16.

13. Guillen-Burrieza, E.; Thomas, R.; Mansoor, B.; Johnson, D.; Hilal, N.; Arafat, H. Effect of dry-out on the fouling of PVDF and PTFE membranes under conditions simulating intermittent seawater membrane distillation (SWMD). J. Membr.Sci. 2013, 438, 126-139. [CrossRef]

14. Li, C.-L.; Wang, D.-M.; Deratani, A.; Quémener, D.; Bouyer, D.; Lai, J.-Y. Insight into the preparation of poly(vinylidene fluoride) membranes by vapor-induced phase separation. J. Membr. Sci. 2010, 361, $154-166$. [CrossRef]

15. Dorrer, C.; Rühe, J. Some thoughts on superhydrophobic wetting. Soft Matter 2009, 5, 51-61. [CrossRef]

16. Feng, X.J.; Jiang, L. Design and Creation of Superwetting/Antiwetting Surfaces. Adv. Mater. 2006, 18, 3063-3078. [CrossRef]

17. Zuo, J.; Chung, T.-S. Metal-Organic Framework-Functionalized Alumina Membranes for Vacuum Membrane Distillation. Water 2016, 8, 586. [CrossRef]

18. Hilal, N.; Ismail, A.F.; Wright, C.J. PVDF Membranes for Membrane Distillation. In Membrane Fabrication; CRC Press: Boca Raton, FL, USA, 2015.

19. Khayet, M.; Matsuura, T. Membrane Distillation: Principles and Applications; Elsevier: Amsterdam, The Netherlands, 2011.

20. Strathmann, H.; Kock, K. The formation mechanism of phase inversion membranes. Desalination 1977, 21, 241-255. [CrossRef]

21. Li, M.; Katsouras, I.; Piliego, C.; Glasser, G.; Lieberwirth, I.; Blom, P.W.; de Leeuw, D.M. Controlling the microstructure of poly(vinylidene-fluoride)(PVDF) thin films for microelectronics. J. Mater. Chem. C 2013, 1, 7695-7702. [CrossRef]

22. Matsuyama, H.; Teramoto, M.; Nakatani, R.; Maki, T. Membrane formation via phase separation induced by penetration of nonsolvent from vapor phase. II. Membrane morphology. J. Appl. Polym. Sci. 1999, 74, 171-178. [CrossRef]

23. Wang, X.; Zhang, L.; Sun, D.; An, Q.; Chen, H. Formation mechanism and crystallization of poly(vinylidene fluoride) membrane via immersion precipitation method. Desalination 2009, 236, 170-178. [CrossRef]

24. Van de Witte, P.; Dijkstra, P.J.; Van den Berg, J.W.A.; Feijen, J. Phase separation processes in polymer solutions in relation to membrane formation. J. Membr. Sci. 1996, 117, 1-31. [CrossRef]

25. Caquineau, H.; Menut, P.; Deratani, A.; Dupuy, C. Influence of the relative humidity on film formation by vapor induced phase separation. Polym. Eng. Sci. 2003, 43, 798-808. [CrossRef]

26. Menut, P.; Su, Y.S.; Chinpa, W.; Pochat-Bohatier, C.; Deratani, A.; Wang, D.M.; Huguet, P.; Kuo, C.Y.; Lai, J.Y.; Dupuy, C. A top surface liquid layer during membrane formation using vapor-induced phase separation (VIPS)—Evidence and mechanism of formation. J. Membr. Sci. 2008, 310, 278-288. [CrossRef]

27. Alkhudhiri, A.; Darwish, N.; Hilal, N. Membrane distillation: A comprehensive review. Desalination 2012, 287, 2-18. [CrossRef]

28. Kang, G.; Cao, Y. Application and modification of poly(vinylidene fluoride) (PVDF) membranes-A review. J. Membr. Sci. 2014, 463, 145-165. [CrossRef]

29. Park, Y.-S.; Lee, C.-K.; Kim, S.-K.; Oh, H.-J.; Lee, S.-H.; Choi, J.-S. Effect of temperature difference on performance of membrane crystallization-based membrane distillation system. Desalin. Water Treat. 2013, 51, 1362-1365. [CrossRef]

(C) 2017 by the authors; licensee MDPI, Basel, Switzerland. This article is an open access article distributed under the terms and conditions of the Creative Commons Attribution (CC BY) license (http:/ / creativecommons.org/licenses/by/4.0/). 